\title{
A straight choice: avoiding septal re-deviation using titanium plates. A 16-year retrospective patient follow-up evaluation*
}

\author{
Jose Miguel Godoy S.', Jose M. Godoy², Andres Godoy³, Carolina Revelo² \\ 'Department of Otolaryngology - Head and Neck Surgery, Clinica las Condes, Santiago, Chile \\ 2 San Diego, CA, USA \\ San Francisco, CA, USA
}

Rhinology 55: 369-375, 2017

https://doi.org/10.4193/Rhino17.020

*Received for publication:

January 25,2017

Accepted: August 7, 2017

\begin{abstract}
Background: To propose a new surgical technique for fixing the nasal septum to the midline, for long term prevention of nasal obstruction, in secondary and select cases of primary septoplasty.
\end{abstract}

Methodology: Retrospective study, within hospital medical center.

Patients: Two hundred and twenty two patients who underwent septoplasty. Data collection occurred consecutively between March 1st of 2000 and May 1st 2016. Twenty six percent females and seventy four percent males.

Main outcome measures: Patterns of septal deformity, materials used for titanium plates, surgical results, symptom improvement, and surgical complications were investigated.

Results: A total of 222 patients were included in this study. 163 patients (73\%) had no previous nasal surgery. Fifty nine patients (27\%) presented with a previous nasal surgery (Table 1). Sixteen year follow up included more than $90 \%$ of patients and resulted in an overall $2.7 \%$ revision rate.

Conclusions: The use of titanium plate for septoplasty has shown to be simple, safe, and easy to learn technique in both secondary and select cases of primary septoplasty. Most importantly the results indicate a long term prevention of the main cause of consultation: nasal obstruction, hence reflecting an effective procedure to add to any otolaryngology practice.

Key words: septoplasty, titanium plates

\section{Introduction}

Septal deviation with or without inferior turbinate hypertrophy, is one of the most common findings in patients with symptomatic nasal obstruction. It follows that septoplasty and inferior turbinate reduction are perhaps the most commonly performed procedures for nasal airway obstruction, accounting for a third of total procedures in otolaryngology.

Despite objective metrics and outcome research becoming increasingly important in the analysis of treatment algorithms, patient selection for septoplasty is frequently based on clinical judgment alone. This is not surprising given that physical examination and various objective measure of nasal airway function do not necessarily correlate with symptoms. Thus, an ideal objective tool for septoplasty evaluation does not exist. Nasal specific questionnaires however, provide a practical approach for outcome assessment, given their focus on symptoms. In this context, both retrospective as well as prospective research have reported septoplasty success rates between 65 and $89 \%^{(1-7)}$. 
It is thus not surprising, given that between $11 \%$ to $35 \%$ of septoplastys' are reported as ineffective (regardless of the surgical technique), that high rates of re-operations occur ${ }^{(8,9)}$.

Moreover, in time, the degree of the benefit in the symptom improvement decays over time. Two thirds of the patients who benefit from surgery, experience symptom recurrence in the coming months post operatively. In Jessen's et al study, this was particularly noticeable as at 9 years post-operatively, only $26 \%$ were symptom-free ${ }^{(10,11)}$.

Multiple components could account for the significant portion of dissatisfied patients, such as surgical technique, inappropriate surgical procedure selection, unrealistic expectations or patient selection amongst others. In this context, we were interested in researching an alternative surgical procedure for septoplasty, which could significantly increase surgical success measured not only as short term symptom improvement, but also one which could sustain long term benefits.

Fixation of the septum using external materials have not been fully explored as opposed to conventional techniques, primarily due to concerns in long term complications such as protrusion of the materials. In addition to what has been published in the literature in recent years, a series of new approaches have emerged to prove the safety and efficacy of titanium materials, such as mini plates or clips to maintain pieces of cartilages together (12-15).

We therefore propose a broader understanding of titanium plates usability and its mechanisms for nasal septum fixation in particular. The purpose of this study is to expose a new fixation method for the nasal septum in patients with deviated septum for secondary revision septoplasty as well as in select cases of primary septoplasty. We present the surgical technique, its proper use, along with a long-term follow-up (sixteen years) of the functional outcomes in a cohort of over two hundred patients.

\section{Materials and methods}

Data collection occurred consecutively between March 1st of 2000 and May 1st 2016. Patients were evaluated and counseled preoperatively, paying particular attention to the objectives of surgery (functional improvement) and possible limitations.

Patients were identified from the operative database. Patients gave informed written consent for publication of clinical and operative information and, in selected cases, for publication of photographs. Accordingly, institutional review board approval was received for this study from the hospital's ethics committee. Radiologic nasal septum imaging was obtained post-operative between 2012 and 2014. From the 2014 cohort onwards we re- quested $\mathrm{x}$-ray control in the operating room in every case before closing the incision.

\section{Surgical technique}

A traditional septoplasty via a hemi-transfixion approach is performed. One of the differences is that the hemi left anterior transfixion incision extends downwards $1 \mathrm{~cm}$ towards the vestibule of the nose, and the incision must be ahead of the anterior edge of the caudal septum. This, in order to place the titanium plate behind the flap, rising from the mucoperichondrium. The attachment of the plate is thus hidden and separated from the nasal incision.

We then carefully lift the mucoperichondrium flap and continue with the incision down towards the nasal spine. The incision needs to be extended further downwards to the vestibule of the nose using a scalpel initially and then with electro cautery, placing a nasal speculum to separate the muscles in the maxilla. Once we approach the superior maxilla we limit the dissection to the edge between the bone and the mucosa of the floor of the nasal cavity. After we have cleared the edge of the nasal cavity floor, we raise the mocuperichondrium up to the nasal spine, and join both flaps. With this initial approach described, we have a clear vision of the nasal septum, the nasal spine, and the upper part of the maxilla close to the floor of the nose (Figure 1). This edge, the limit between nasal floor mucosa and maxilla is very important as a guide for the attachment of the plate, which is accomplished using two screws, 3 to $4 \mathrm{~mm}$ below this described margin (Figure 2).

We continue by raising a mucoperichondrium flap on the opposite side, large enough to pass the other arm of the T plate once folded to be inserted on the opposite side of the septum, holding the nasal septum at both sides with a piece of titanium plate (as if pressing the septum in a sandwich form) (Figure 3), providing a convenient and straightforward method to mold at our suitability and consequently fix the deviated septum with our plate (Figures 6 and 7).

If the nasal spine is too prominent we can cut a small portion of bone, and therefore, we can approximate the plate to the spine and septum adding a steady attachment to it.

Fixation of the nasal septum begins with a simple $T$ shaped plate (titanium plate, regular, $7 \mathrm{~mm} \times 7 \mathrm{~mm}, 27 \mathrm{~mm}$ horizontal $\times 30$ $\mathrm{mm}$ vertical, with $0.6 \mathrm{~mm}$ of thickness to fix with self-drilling screws of $1.5 \mathrm{~mm}$ diameter and $5 \mathrm{~mm}$ length, by Zymmer-Biomet ex Walter Lorenz Surgical, INC) used in cranial facial fractures, neurosurgery and maxillofacial surgery. The titanium plates are then placed in the direction of the deviation, to prevent the septum returning to the same direction (Figure 2). 


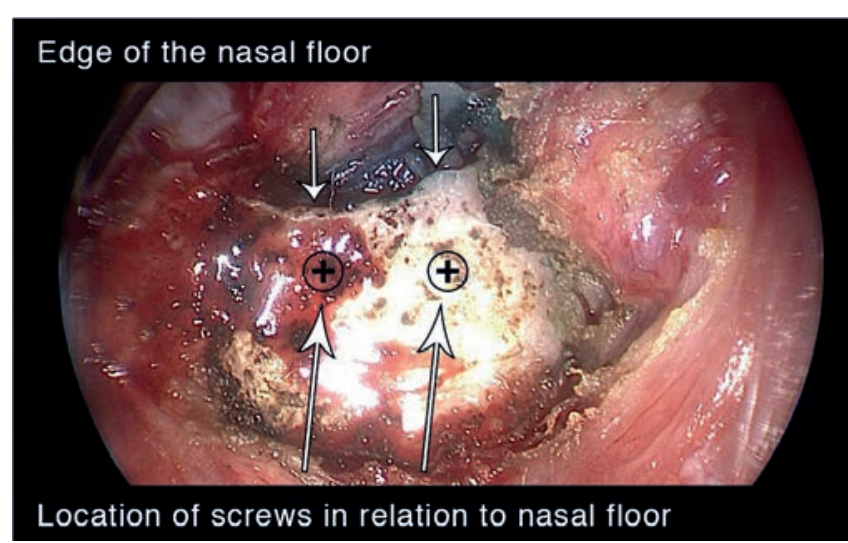

Figure 1. Position of titanium plate.

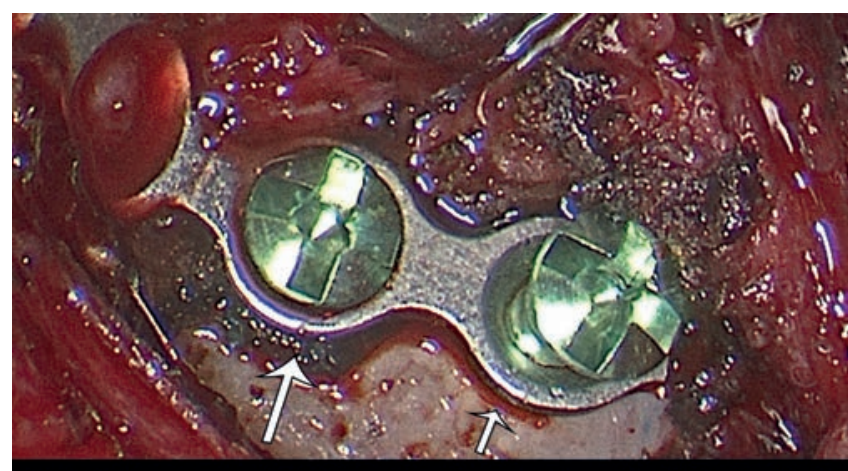

Self drilling screws of $1.5 \mathrm{~mm}$ diameter and $5 \mathrm{~mm}$ length being fixed

Figure 2. The plate with 2 screws attached to the maxilla.

The plates are fixed over the maxilla, below the limit of the nasal floor in a distance about $3 \mathrm{~mm}$ to $4 \mathrm{~mm}$ using two titanium screws ( $5 \mathrm{~mm}$ in length) and the limit of the nasal floor (thus preventing approaching the dental roots). We can add a couple of stitches with nylon 4-0 between the plate and the nasal septum in order to reassure that it is attached firmly and in the correct place (Figures 2, 4, and 5). In select cases, we take a small bite of the septum where the plate is bended so that the bended section of the plate remains inside the septum (Figure 4). Lately, we have been using a small rectangular piece of cartilage, taken from the septum, which we suture at the site where the plate is bended (upper section) to protect this area from possible extrusions due to trauma (Figure 5).

With our expertise during these years we highly recommend the of use X-ray in the operating room while the patient is still under anesthesia in order to verify the correct placement of the plate in the midline, which can be accommodated and adjusted if necessary to the midline before closure of the incision. $A$ couple of transfixed sutures are placed to maintain the mucosa attached to the septum and the plate to reduce the chances of hematoma. Finally, nasal packing (Merocel PVA, Medtronic, USA) were placed for an average of 2 days on the outside in the nasal

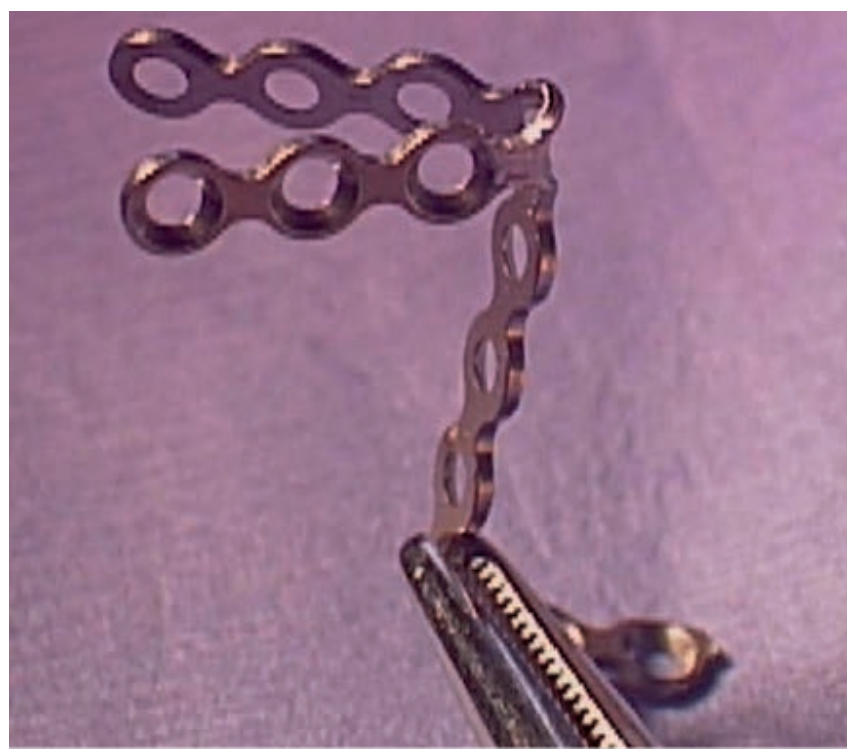

Figure 3. The T shape titanium is folded before insertion and fixed to the maxilla.

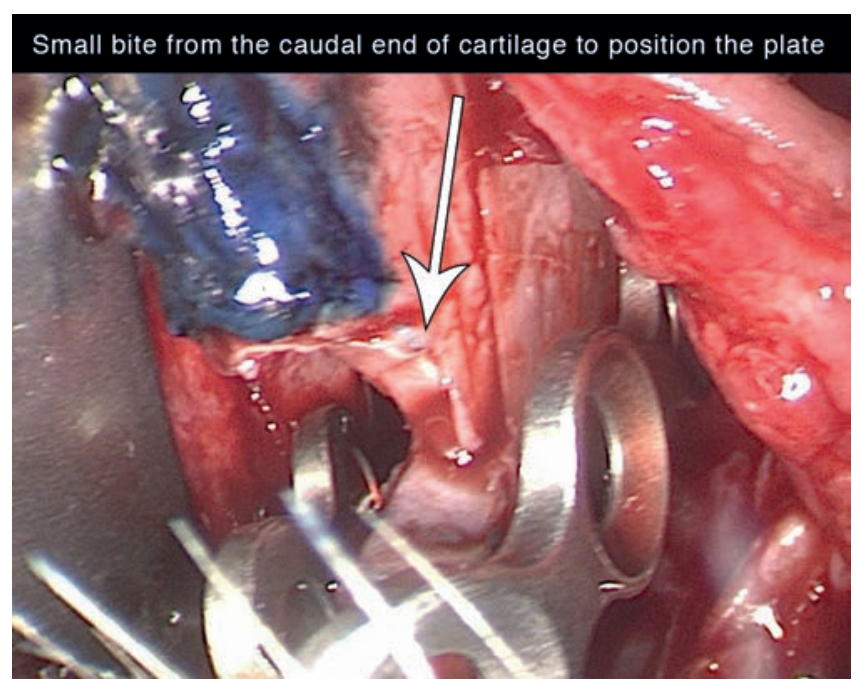

Figure 4. A small bite removed from the septum to accommodate the plate.

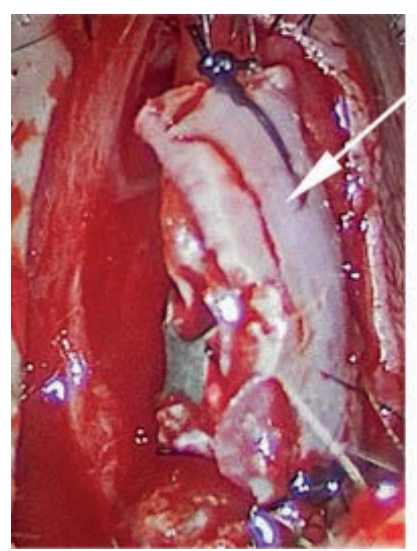

\section{Cartilage graft from septum covering the site of where the plate is bended.}

Figure 5. A small and thin piece of nasal cartilage to protect the upper part of the plate. 


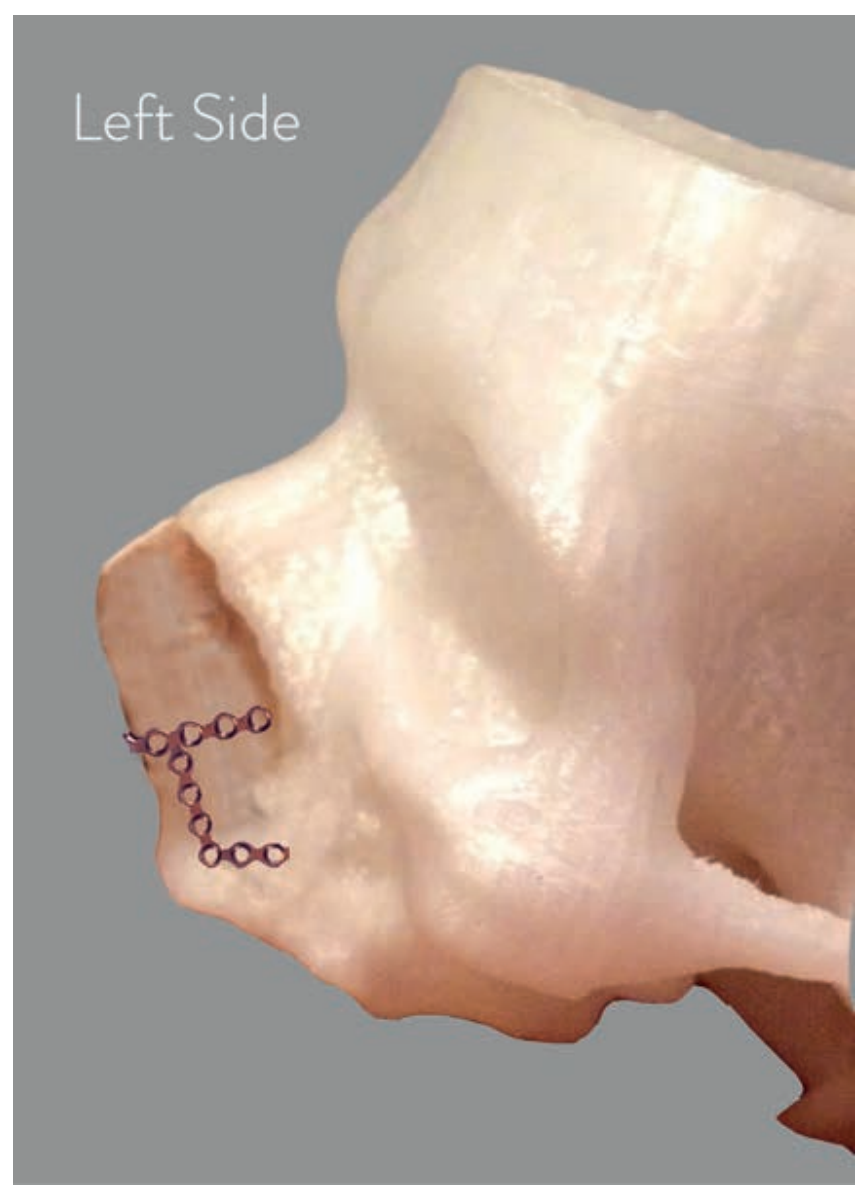

Figure 6. 3D model showing left side titanium plate placement.

vestibule.

\section{Postoperative management}

Assuming that no complications are encountered, nasal packing is removed after twenty-four to forty-eight hours. Some patients experience a slight edema of the left upper lip that typically resolve within a few days. If turbinates are not operated, we recommend to follow up once a year and if turbinates are partially removed, the recommended follow up is extended until no crusts are in the nasal cavity. Most patients are subsequently seen every 12 months or if they are exposed to trauma to the nose.

\section{Titanium plate selection}

After a few years of utilizing a straight plate, we decided to implement a T plate structure which consists in folding the T-plate (titanium plate), passing one arm over the other side of the septum and placing the septum in the middle; therefore, folding both arms together fixing it at a midline position (Figure 3). The remainder of the nasal septum is the same length as the titanium plate attached to it. In other words, the nasal cartilage is resected to be the same length as the titanium arms that will

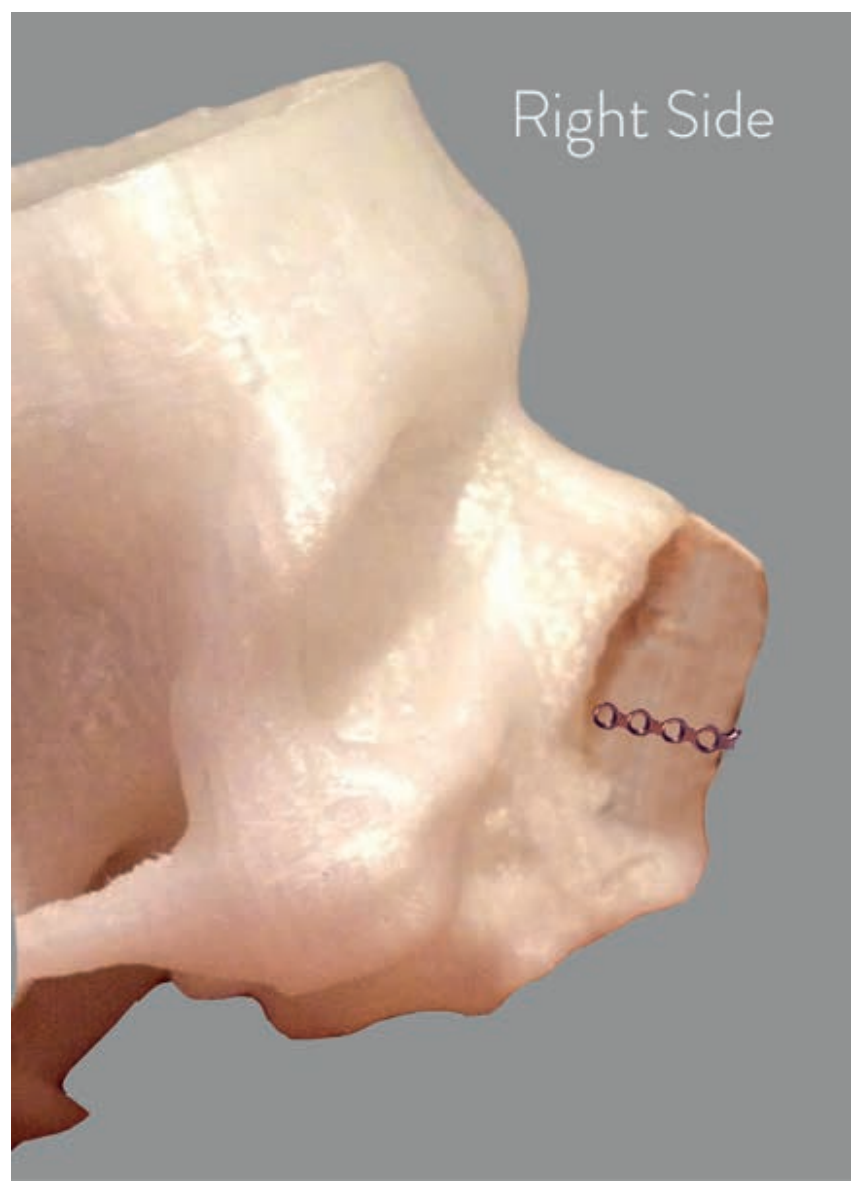

Figure 7. 3D model showing right side titanium plate placement.

be fixing the septum together. We have learned that the plate must be placed a few millimeters behind the caudal border of the inside of the cartilage so that the columella can move freely (Figures 6 and 7).

\section{Results}

A total of 222 patients were included in this study. 163 patients (73\%) had no previous nasal surgery. 59 (27\%) presented with a previous nasal surgery (Table 1). Sixteen year follow up included more than $90 \%$ of patients.

As for complications, two patients presented dysesthesia due to the initial technique used, in which the plate was placed closer to the dental roots. Three patients suffered extrusion of a portion of the plate secondary to an inappropriate initial technique. Regarding the second period of the technique (as commented in MATERIALS AND METHODS), one patient experienced local fibrosis in the higher part of the septum, requiring removal of the titanium plate and the fibrosis. We did not encounter any of the initial dysesthesia and extrusion issues, yet we encountered three cases of incorrect alignment. For this reason, we implemented intra-operative $x$-rays. 
Table 1. Patient cohort characteristics and results.

\begin{tabular}{|lc|}
\hline & number \\
\hline Total & 222 \\
\hline Mean Age (years) & 32 \\
\hline Gender & $58(26)$ \\
\hline Female (\%) & $164(74)$ \\
\hline Male (\%) & \\
\hline History & $59(27)$ \\
\hline \multicolumn{1}{|l}{ Previous septoplasty (\%) } & $163(73)$ \\
\hline No previous septoplasty (\%) & $9(4)$ \\
\hline Complications (\%) & \\
\hline First Period of Technique (2000-2004): & $3(1.3)$ \\
\hline $\begin{array}{l}\text { Extrusion due to inappropriate } \\
\text { technique (\%) }\end{array}$ & $0(0)$ \\
\hline Incorrect Alignment (\%) & $2(0.9)$ \\
\hline $\begin{array}{l}\text { Dental Disesthesias (\%) } \\
\text { Second Period of Technique (2004-2016): }\end{array}$ & $0(0)$ \\
\hline $\begin{array}{l}\text { Extrusion due to inappropriate } \\
\text { technique (\%) }\end{array}$ & $0(0)$ \\
\hline Incorrect Alignment (\%) & $3(1.3)$ \\
\hline Dental Disesthesias (\%) & $0.47)$ \\
\hline Local fibrosis (\%) & \\
\hline
\end{tabular}

\section{Discussion}

For the past 16 years, we have been learning how to fix the septum with a titanium plate. We have corrected our own errors, and now introduce a technique which we feel very confident of the outcomes achieved with our patients.

Multiples techniques have been used for the caudal treatment of septal deviations and the deviated septum. Many of these traditional techniques result in a brief improvement followed by a deterioration of the clinical symptoms ${ }^{(16)}$. Hence, it was of paramount importance to us to propose a new technique which could result in the definitive improvement of the symptoms while reflecting low post-operative complications.

Recently, literature has published the use of a polydioxanone plate as an absorbable scaffold ${ }^{(17)}$. However, this material in order to support the re-implanted cartilage, is not fixed to a rigid structure for support in order to avoid the possible reaccommodation of the septum once the scaffold is reabsorbed. Therefore, in these cases, the septum can possibly move and re-deviated again.
Initial experience began only considering operating on patients who had more than one previous surgery (septoplasty) and explicitly requested a new approach. Despite extensive literature showing that titanium has been used as a safe material for severe head and neck trauma to the nose or facial structures, our initially approach was cautious ${ }^{(8)}$. We implemented the technique with a select and limited cohort patients over a five year time-frame, in order to gain data on the plates safety and on the technique's overall effectiveness. We then continued with patients that had not had previous septal surgery, but which expressed symptoms of nasal obstruction, secondary to deviation and completely lateralized septum to either side.

In regard to the initial phase, our learning curve began with a thick titanium plate, hard to mold and adjust for the correction of caudal deviated septum, performing only a few cases. However, within our second stage, once we had a more malleable plate, it was easier to place, mold, and attach it to the septum. We also improved the way the plate was attached to the maxillary bone in order to avoid the paresthesias we experienced. Thus we began fixing the plate during the last three years near to the nasal floor, eliminating any type of dental-paresthesia complications and secondary dental sensitivity adverse effect.

Due to the form and the structure of the plates, the ideal recommendations is placing it to an angle of thirty degrees in a relation with that to be held. This follows an engineers and architectural common principle. However, we try to maintain the plate in close proximity to the septum in order to prevent false pouches of mucosa unattached to the septum. We managed to reduce the complaints from patients regarding the sensitivity of the teeth and the possibility of primary extrusion by the plate itself by only attaching it to the septum. With the correct training, the time to place the titanium plate is an average of five to ten minutes, starting from molding, bending and fixing it to the septum with assurance of the correct position.

This particular procedure (adding the titanium plate) does not significantly increase the operation time, yet it provides the reassurance that if there is a trauma to the nose, and depending of the severity, the plate can absorb the forces of the trauma, allowing the septum to be kept in place. Also, it reassures a surgeon that if there is no trauma, the septum will be kept in midline for life. This favors the use of the plate considering previous literature that describes that for conventional septoplasty, 11\% to $35 \%$ are reported as ineffective ${ }^{(8,9)}$. In an extensive 10 -year retrospective study undertaken in a tertiary care otorhinolaryngology center by Dinis et al ${ }^{(18)}$, a significant portion of patients reported a poor to mediocre result (23\%).

If we specifically look at revision rates within the subset of unsa- 
tisfied patients, a study of 2,168 septoplasties by any technique, showed a revision rate of $3.2 \%$ among experienced surgeons ${ }^{(19)}$. More recently, and in line with the above revision rate, a study by Spataro et al revealed that 5,389 of 172,324 patients (3.1\%) required revision ${ }^{(20)}$. Studies focusing on specific septoplasty techniques showed higher revision rates of $7 \%$ to $8 \%{ }^{(21,22)}$. The proposed technique using titanium plates, reflected an overall $4 \%$ complication rate, of which, $2.7 \%$ required revision. Of note, there may be an under-estimation of the revision rates reported in the literature because the studies mentioned do not include patients who seek revision surgery at another institution, thereby lowering the reported revision percentages.

Extrusion is a common concern when utilizing external materials. Our extrusion rate of $1.3 \%$ is lower than what the literature reflects in terms of traditional septoplasty revision rates. Our extrusion rate has been mainly due to to an inappropriate placement and fixing of the plate during the first few years of experience. Thus, within the second period of our technique, our cluster of patients reflected a zero percent extrusion rate.

During the second period of our technique, we encountered three cases ( $1.3 \%$ of complications overall) of misaligned plates during the post-operative check-ups. These patients required minimal anesthesia and via a manual adjustment procedure of the plate, we were able to correctly re-align the plate. We therefore implemented, from 2014 onwards, intra-operative x-rays before closure. Utilizing a portable imaging system, this additional step has provided a final safety check-point which allows us to verify the correct placement and alignment of the plate. Post implementation of intra-operative $x$-rays, we encountered three cases in which we modified the alignment before finalizing the surgery. It is our recommendation that intra-operative imaging is a quick procedure which can provide a final safety check-point as well as an objective metric of surgical competency.

The proposed technique increases the chance of long term surgical success as measured by resolved nasal obstruction. In an anterior deviation, there is a much higher risk of a new deviation of the septum, when not using a titanium plate fixation methodology than compared to the possibility of extrusion when fixing the septum with a titanium plate. In a study by Gillman et al ${ }^{(23)}$, persistent structural septal deviation was noted as the most frequent characteristic in revision septoplasty.

In addition, the plate does not add thickness to the cartilage as others graft or scaffolding materials because it is only $0.6 \mathrm{~mm}$ thick, allowing for a sustained patent airway. Most importantly, symptom improvement is achieved and maintained. After two months, it is difficult to palpate the plate.
It is our responsibility to utilize health care resources accountably. Evidence-based treatment approaches are increasingly important, and our sixteen year follow up cohort data support the investment of this methodology over traditional surgical techniques. These findings highlight the importance of maximizing surgical efforts to improve function in the context of long term effects.

We feel confident about the implementation of this technique and the impact it can produce in society. Our goal is to provide hope to many patients that were lacking an adequate surgical technique, assuring them that the plate and septum will be kept in midline for life. Most importantly the results indicate a long-term prevention of the main cause of consultation: nasal obstruction, resulting in a permanent functional improvement. We advocate the potential development of a plate specialized for this purpose being longer and with more arms in order to enhance the support towards the upper and lower portion of the septum.

We can recommend that every septum be attached to a titanium plate in order to assure a good result thus not allowing the forces of the nasal cartilage to overcome the correction, especially in those cases where we removed a portion that is luxated at the floor of the nose in order to center the septum and also is detached from the perpendicular plate of the etmoid in order to correct the deviation of the septum. The plate has shown in time to be able to absorb the forces of stress and trauma without new deviations of the septum.

\section{Conclusion}

This technique has shown to be simple, safe, easy to learn, and most importantly assures prevention of patients main complains of a deviated septum which are: nasal obstruction, providing them with an increased quality of life and fostering new possibilities for re operated patients. We hope that in the future with a customized titanium plate design, we can lower the risk of extrusion to less than one percent.

\section{Authorship contribution}

JMGS.: Participated in the conception and study design, as well as: data collection, data analysis and interpretation, drafting of the article, and finally in critical revision of the article.

JMG: Participated in the conception and study design, as well as: drafting of the article, and finally in critical revision of the article. AG: Participated in the conception and study design, as well as: data collection, data analysis and interpretation, drafting of the article, and finally in critical revision of the article.

CR: Participated in the study data collection, data analysis and interpretation. 


\section{Conflict of interest}

No financial interest and no conflict of interest.

\section{References}

1. Siegel NS, Gliklich RE, Taghizadeh F, Chang Y. Outcomes of septoplasty. Otolaryngol Head Neck Surg. 2000; 122: 228-232.

2. Holmstrom M, Kumlien J. A clinical follow up of septal surgery with special attention to the value of preoperative rhinomanometric examination in the decision concerning operation. Clin. Otolaryngol. Allied Sci. 1988; 13: 115-120.

3. Gandomi B, Bayat A, Kazemei T. Outcomes of septoplasty in young adults: the Nasal Obstruction Septoplasty Effectiveness study. Am J Otolaryngol. 2010; 31: 189-192.

4. Pirila T, Tikanto J. Unilateral and bilateral effects of nasal septum surgery demonstrated with acoustic rhinometry, rhinomanometry, and subjective assessment. Am J Rhinol. 2001; 15: 127-33.

5. Samad I, Stevens HE, Maloney A. The efficacy of nasal septal surgery. J Otolaryngol. 1992; 21: 88-91.

6. Stewart MG, Smith TL, Weaver EM, et al. Outcomes after nasal septoplasty: results from the Nasal Obstruction Septoplasty Effectiveness (NOSE) study. Otolaryngol Head Neck Surg. 2004; 130: 283-290.

7. Uppal S, Mistry H, Nadig S, Back G, Coatesworth A. Evaluation of patient benefit from nasal septal surgery for nasal obstruction. Auris Nasus Larynx. 2005; 32: 129-137.

8. Sundh C, Sunnergren O. Long-term symptom relief after septoplasty. Eur Arch Otorhinolaryngol. 2015; 272(10): 2871-2875.

9. Bitzer EM, Dorning H, Schwartz FW. Clinical success of surgical correction of the nasal septum. Laryngorhinootologie. 1996; 75(11): 649-656; discussion 656-649.

10. Ho WK, Yuen AP, Tang KC, Wei WI, Lam PK.
Time course in the relief of nasal blockage after septal and turbinate surgery. Arch Otolaryngol Head Neck Surg. 2004; 130: 324-32.

11. Jessen M, Ivarsson A, Malm L. Nasal airway resistance and symptoms after functional septoplasty: comparison of findings at 9 months and 9 years. Clin. Otolaryngol. Allied Sci 1989;14: 231-234.

12. Manuel A, Fishman JM, Majumdar A, et al. Titanium mini-plate for securing septal cartilage a case of high-impact nasal trauma. Ann R Coll Surg Engl. 2009; 91(5):433-434.

13. Manavbasi Yl, Kerem H, Erdem A. The use of titanium clips in septal surgery for correction and strengthening. J Plast Reconstr Aesthet Surg. 2012; 65(6): 739-746.

14. Maniglia AJ, Swim S. Parietal bone graft and titanium plate fixation in nasal reconstruction. Laryngoscope. 1993; 103(9): 10661072.

15. Ahn MS, Monhian N, Maas CS, et al. Total nasal reconstruction with alloplastic and autogenous grafts. Facial Plast Surg. 1998; 14(2): 145-150.

16. Gandomi B, Bayat A, Kazemei T. Outcomes of septoplasty in young adults: the Nasal Obstruction Septoplasty Effectiveness study. Am J Otolaryngol. 2010; 31(3): 189192.

17. Rimmer J, Ferguson LM, Saleh HA. Versatile applications of the polydioxanone plate in rhinoplasty and septal surgery. Arch Facial Plast Surg. 2012; 14(5): 323-330.

18. Dinis PB, Haider H. Septoplasty: Long term evaluation of results. Am J Otolaryngol 2002; 23: 85-90.

19. KarlssonTR, ShakeelM, Al-AdhamiA, Suhailees, Ram B, Ah-See KW. Revision nasal surgery after septoplasty: trainees versus trainers. Eur Arch Otorhinolaryngol. 2013; 270(12): 3063-3067.

20. Emily Spataro, MD; Jay F. Piccirillo, MD; Dorina Kallogjeri, MD, MPH; Gregory $\mathrm{H}$. Branham, MD; Shaun C. Desai, MD Revision Rates and Risk Factors of 175842 Patients Undergoing Septorhinoplasty 2016; 18(3): 212-9.

21. GubischW. Twenty-five years experiencewith extracorporeal septoplasty. Facial Plast Surg. 2006; 22(4): 230-239.

22. SedwickJD, LopezAB, GajewskiBJ, SimonsRL. Caudal septoplasty for treatment of septal deviation: aesthetic and functional correction of the nasal base. Arch Facial Plast Surg. 2005; 7(3): 158-162.

23. Gillman GS, Egloff AM, Rivera-Serrano CM. Revision septoplasty: a prospective diseasespecific outcome study. Laryngoscope. 2014; 124(6): 1290-1295.

Jose Miguel Godoy S., MD

Department of Otolaryngology

Head \& Neck Surgery

Clinica las Condes

Santiago

Chile

Tel: +56990202209

E-mail:jmgodoy@clc.cl 